\title{
Multi-objective optimization of the turning process using a Gravitational Search Algorithm (GSA) and NSGA-II approach
}

\author{
Klancnik, S. ${ }^{a,}{ }^{*}$, Hrelja, M. ${ }^{\mathrm{a}, \mathrm{b}}$, Balic, J. ${ }^{\mathrm{a}}$, Brezocnik, M. ${ }^{\mathrm{a}}$ \\ ${ }^{a}$ Production Engineering Institute, Faculty of Mechanical Engineering, University of Maribor, Maribor, Slovenia \\ ${ }^{b}$ AVL, Piezocryst: Advanced Sensorics GmbH, Graz, Austria
}

\section{A B S T R A C T}

In this paper, we proposed a Gravitational Search Algorithm (GSA) and an NSGA-II approach for multi-objective optimization of the CNC turning process. The GSA is a swarm intelligence method exploiting the Newtonian laws on elementary mass objects interaction in the search space. The NSGA-II is an evolutionary algorithm based on non-dominated sorting. On the basis of varying values of the three independent input machining parameters (i.e., cutting speed, depth of cut, and feed rate), the values of the three dependent output variables were measured (i.e., surface roughness, cutting forces, and tool life). The obtained data set was divided further into two subsets, for the training data and the testing data. In the first step of the proposed approach, the GSA and the training data set were applied to modelling a suitable model for each output variable. Then the accuracies of the models were checked by the testing data set. In the second step, the obtained models were used as the objective functions for a multi-objective optimization of the turning process by the NSGA-II. The optimization constraints relating to intervals of dependent and independent variables were set on the theoretical calculations and confirmed with experimental measurements. The goal of the multi-objective optimization was to achieve optimal surface roughness, cutting forces, and increasing of the tool life, which reduces production costs. The research has shown that the proposed integrated GSA and NSGA-II approach can be implemented successfully, not only for modelling and optimization of the CNC turning process, but also for many other manufacturing processes.
\end{abstract}

\section{ARTICLE INFO}

Keywords:

Turning

Multi-objective optimization

Evolutionary algorithms

Particle swarm

Gravitational Search Algorithm

NSGA-II algorithm

*Corresponding author:

simon.klancnik@um.si

(Klancnik, S.)

Article history:

Received 8 July 2016

Revised 19 November 2016

Accepted 21 November 2016

\section{References}

[1] Hrelja, M., Klancnik S., Balic J., Brezocnik M. (2014). Modelling of a turning process using the gravitational search algorithm, International Journal of Simulation Modelling, Vol. 13. No. 1, 30-41, doi: 10.2507/IJSIMM13(1)3.248.

[2] Lestan, Z., Klancnik, S., Balic, J., Brezocnik, M. (2015). Modeling and design of experiments of laser cladding process by genetic programming and nondominated sorting, Materials and Manufacturing Processes, Vol. 30, No. 4, 458-463, doi: 10.1080/10426914.2014.973586.

[3] Ficko, M., Palcic, I. (2013). Designing a layout using the modified triangle method, and genetic algorithms, International Journal of Simulation Modelling, Vol. 12, No. 3, 237-251, doi: 10.2507/IJSIMM12(4)3.244.

[4] Alberti, M., Ciurana, J., Casadesús, M. (2005). A system for optimising cutting parameters when planning milling operations in high-speed machining, Journal of Materials Processing Technology, Vol. 168, No. 1, 25-35, doi: 10.1016/i.jmatprotec.2004.09.092.

[5] Bharathi Raja, S., Baskar, N. (2011). Particle swarm optimization technique for determining optimal machining parameters of different work piece materials in turning operation, The International Journal of Advanced Manufacturing Technology, Vol. 54, No. 5, 445-463, doi: 10.1007/s00170-010-2958-y. 
[6] Zain, A.M., Haron, H., Sharif, S. (2011). Optimization of process parameters in the abrasive waterjet machining using integrated SA-GA, Applied Soft Computing, Vol. 11, No. 8, 5350-5359, https://doi.org/10.1016/i.asoc. 2011.05.024.

[7] Billatos, S.B., Tseng, P.-C. (1991). Knowledge-based optimization for intelligent machining, Journal of Manufacturing Systems, Vol. 10, No. 6, 464-475, doi: 10.1016/0278-6125(91)90004-L.

[8] Byrne, G., Dornfeld, D., Inasaki, I., Ketteler, G., König, W., Teti, R. (1995). Tool condition monitoring (TCM) - The status of research and industrial application, CIRP Annals - Manufacturing Technology, Vol. 44, No. 2, 541-567, doi: 10.1016/S0007-8506(07)60503-4.

[9] Chua, M.S., Rahman, M., Wong, Y.S., Loh, H.T. (1993). Determination of optimal cutting conditions using design of experiments and optimization techniques, International Journal of Machine Tools and Manufacture, Vol. 33, No. 2, 297-305, doi: 10.1016/0890-6955(93)90081-5.

[10] Yusup, N., Zain, A.M., Hashim, S.Z.M. (2012). Evolutionary techniques in optimizing machining parameters: Review and recent applications (2007-2011), Expert Systems with Applications, Vol. 39, No. 10, 9909-9927, doi: 10.1016/i.eswa.2012.02.109.

[11] Zain, A.M., Haron, H., Sharif, S. (2010). Application of GA to optimize cutting conditions for minimizing surface roughness in end milling machining process, Expert Systems with Applications, Vol. 37, No. 6, 4650-4659, doi: 10.1016/i.eswa.2009.12.043.

[12] D'Addona, D.M., Teti, R. (2013). Genetic algorithm-based optimization of cutting parameters in turning processes, Procedia CIRP, Vol. 7, 323-328, doi: 10.1016/i.procir.2013.05.055.

[13] Khaider, B., Asma, T. (2016). Hard turning behavior improvement using NSGA-II and PSO-NN hybrid model, The International Journal of Advanced Manufacturing Technology, Vol. 86, No. 9, 3527-3546, doi: 10.1007/s00170016-8479-6.

[14] Krimpenis, A., Vosniakos, G.-C. (2009). Rough milling optimisation for parts with sculptured surfaces using genetic algorithms in a Stackelberg game, Journal of Intelligent Manufacturing, Vol. 20, No. 4, 447-461, doi: 10.1007/s10845-008-0147-8.

[15] Suresh, P.V.S., Venkateswara Rao, P., Deshmukh, S.G. (2002). A genetic algorithmic approach for optimization of surface roughness prediction model, International Journal of Machine Tools and Manufacture, Vol. 42, No. 6. 675680, doi: 10.1016/S0890-6955(02)00005-6.

[16] Mizugaki, Y., Hao, M., Sakamoto, M., Makino, H. (1994). Optimal tool selection based on genetic algorithm in a geometric cutting simulation, CIRP Annals - Manufacturing Technology, Vol. 43, No. 1, 433-436, doi: doi:10.1016/ S0007-8506(07)62247-1.

[17] Jurkovic, Z., Cukor, G., Brezocnik, M., Brajkovic, T. (2016). A comparison of machine learning methods for cutting parameters prediction in high speed turning process, Journal of Intelligent Manufacturing, (in Press), doi: 10.1007/s10845-016-1206-1.

[18] Cukor, G., Jurkovic, Z. (2010). Optimization of turning using evolutionary algorithms, Engineering Review, Vol. 30, No. 2, 1-10.

[19] Mocnik, D., Paulic, M., Klancnik, S., Balic, J. (2014). Prediction of dimensional deviation of workpiece using regression, ANN and PSO models in turning operation, Tehnički vjesnik - Technical Gazette, Vol. 21, No. 1, 55-62.

[20] El-Mounayri, H., Kishawy, H., Briceno, J. (2005). Optimization of CNC ball end milling: A neural network-based model, Journal of Materials Processing Technology, Vol. 166, No. 1, 50-62, doi: 10.1016/i.jmatprotec.2004.07.097.

[21] Muñoz-Escalona, P., Maropoulos, P.G. (2010). Artificial neural networks for surface roughness prediction when face milling Al 7075-T7351, Journal of Materials Engineering and Performance, Vol. 19, No. 2, 185-193, doi: 10.1007/s11665-009-9452-4.

[22] Zhang, Y., Gong, D.-W., Ding, Z.-H. (2011). Handling multi-objective optimization problems with a multi-swarm cooperative particle swarm optimizer, Expert Systems with Applications, Vol. 38, No. 11, 13933-13941, doi: 10.1016/j.eswa.2011.04.200.

[23] Chiang, F., Braun, R., Agbinya, J.I. (2007). Self-configuration of network services with biologically inspired learning and adaptation, Journal of Network and Systems Management, Vol. 15, No. 1, 87-87, doi: 10.1007/s10922006-9056-3.

[24] Rashedi, E., Nezamabadi-pour, H., Saryazdi, S. (2009). GSA: A gravitational search algorithm, Information Sciences, Vol. 179, No. 13, 2232-2248, doi: 10.1016/j.ins.2009.03.004.

[25] Jurkovic, Z. (2007). Modeliranje i optimizacija parametara obrade primjenom evolucijskih algoritama kod inteligentnih obradnih sustava, PhD thesis, University of Rijeka, Rijeka, Croatia.

[26] Wang, L., Wang, T.-G., Luo, Y. (2011). Improved non-dominated sorting genetic algorithm (NSGA)-II in multiobjective optimization studies of wind turbine blades, Applied Mathematics and Mechanics, Vol. 32, No. 6, 739748, doi: $10.1007 / \mathrm{s} 10483-011-1453-\mathrm{x}$. 


\title{
Multi-objective optimization of the turning process using a Gravitational Search Algorithm (GSA) and NSGA-II approach
}

\author{
Klancnik, S. ${ }^{a,}{ }^{*}$, Hrelja, M. ${ }^{\mathrm{a}, \mathrm{b}}$, Balic, J. ${ }^{\mathrm{a}}$, Brezocnik, M. ${ }^{\mathrm{a}}$ \\ ${ }^{a}$ Production Engineering Institute, Faculty of Mechanical Engineering, University of Maribor, Maribor, Slovenia \\ ${ }^{\mathrm{b}} \mathrm{AVL}$, Piezocryst: Advanced Sensorics GmbH, Graz, Austria
}

\begin{abstract}
POVZETEK
$\mathrm{V}$ članku smo predlagali gravitacijski iskalni algoritem (angl. GSA) in metodo NSGA-II za večkriterijsko optimiranje postopka CNC struženja. GSA je metoda roja delcev, ki izkorišča Newtonske gravitacijske zakone oziroma interakcije med elementarnimi masnimi delci v iskalnem prostoru. Metoda NSGA-II je evolucijski algoritem, ki temelji na nedominiranem razvrščanju. Na osnovi spreminjanja vrednosti treh neodvisnih vhodnih spremenljivk (obdelovalne hitrosti, globine reza in podajanja) smo dobili vrednosti treh odvisnih izhodnih spremenljivk (hrapavosti površine, odrezovalne sile in življenske dobe orodja). Dobljene podatke smo razdelili $\mathrm{v}$ dve skupini, in sicer $\mathrm{v}$ skupino za učenje in skupino za testiranje. V prvem koraku predlaganega pristopa smo uporabili metodo GSA in podatke za učenje; tako smo dobili ustrezen model za vsako izhodno spremenljivko. Nato smo s pomočjo podatkov za testiranje preverili uspešnost učenja. V drugem koraku smo doblejne modele uporabili kot kriterijske funkcije za večkriterijsko optimizacijo postopka struženja z algoritmom NSGA-II. Omejitve, ki se nanašajo na intervale odvisnih in neodvisnih spremenljivk smo dobili na osnovi teoretičnih izračunov, ki smo jih potrdili tudi z meritvami. Namen večkriterijske optimizacije je bil doseči optimalne površinsko hrapavost in odrezovalne sile, a hkrati povečati življensko dobo orodja ter tako zmanjšati izdelovalne stroške. Raziskava je pokazala, da predlagan povezan GSA in NSGA-II pristop ne omogoča le uspešnega modeliranja in optimiranja postopka CNC struženja, ampak tudi mnogih preostalih obdelovalnih postopkov.
\end{abstract}

\section{PODATKI O ČLANKU}

Ključne besede:

Struženje

Večkriterijska optimizacija

Evolucijski algoritmi

Roj delcev

Gravitacijski iskalni algoritem

NSGA-II algoritem

*Kontaktna oseba: simon.klancnik@um.si

(Klancnik, S.)

Zgodovina članka:

Prejet 8. julija 2016

Popravljen 19. novembra 2016

Sprejet 21. novembra 2016 